\title{
Prognostic Significance of the Double Pressure Reserve in Patients with Chronic Heart Failure
}

\author{
Lee Ingle*,1 and Andrew Clark ${ }^{2}$ \\ ${ }^{I}$ Carnegie Research Institute, Leeds Metropolitan University, Becketts Park, Headingley, Leeds LS6 3QS, UK \\ ${ }^{2}$ Department of Cardiology, University of Hull, Castle Hill Hospital, Cottingham, Kingston-upon-Hull HU16 5JQ, UK
}

\begin{abstract}
Introduction: The double pressure reserve (DPR) has recently been shown to have greater prognostic power than metabolic equivalents, heart rate indices, and systolic blood pressure in healthy subjects. It is unclear whether DPR offers any prognostic value in a heart failure population where variables derived from metabolic gas exchange data provide important prognostic information.

Methods: Patients underwent a symptom-limited, treadmill-based exercise test with metabolic gas exchange measurements using the modified Bruce protocol. DPR was calculated as the product of peak systolic blood pressure and peak heart rate subtracted from the product of resting systolic blood pressure and resting heart rate values.

Results: 363 patients (mean $\pm \mathrm{SD}$; age $74 \pm 11$ years; $81 \%$ males; left ventricular ejection fraction $34 \pm 6 \%$; peak $\mathrm{VO}_{2} 19.0 \pm$ $5.1 \mathrm{~mL} \cdot \mathrm{kg}^{-1} \cdot \mathrm{min}^{-1} ; \mathrm{VE} / \mathrm{VCO}_{2}$ slope $37 \pm 9$; double pressure reserve $10,510 \pm 6,046 \mathrm{mmHg} \cdot \mathrm{beat}^{-1}$ ) were included in the study. Peak $\mathrm{VO}_{2}$ (hazard ratio $(\mathrm{HR})=0.87 ; P<0.0001,95 \%$ confidence intervals $(\mathrm{CI})=0.75-0.99$ ), $\mathrm{VE} / \mathrm{VCO} 2$ slope $(\mathrm{HR}=1.03 ; P=0.04 ; 95 \% \mathrm{CI}=1.00-1.06)$, and age $(\mathrm{HR}=1.02 ; P=0.09 ; 95 \% \mathrm{CI}=0.98-1.05)$ were the strongest independent predictors of mortality. DPR was not a univariate predictor of mortality $(P=0.7 ; \mathrm{HR}=1.0 ; 95 \% \mathrm{CI}=0.99-1.0)$.

Conclusion: DPR does not predict mortality in patients with CHF. Traditional prognostic markers derived from metabolic gas exchange including peak $\mathrm{VO}_{2}$ and the $\mathrm{VE} / \mathrm{VCO}_{2}$ slope are more important.
\end{abstract}

Keywords: Systolic blood pressure, heart rate, CPET, ventilation, mortality.

\section{INTRODUCTION}

Cardiopulmonary exercise testing (CPET) with metabolic gas exchange is a standard test for predicting survival in patients with chronic heart failure (CHF). CHF patients have an impaired response to exercise as shown by a lower peak oxygen uptake [1] and anaerobic threshold than healthy subjects [2]. Patients with $\mathrm{CHF}$ also have an increase in the slope relating ventilation (VE) to carbon dioxide production $\left(\mathrm{VCO}_{2}\right)$, which is also related to survival $[3,4]$.

Recently, Rafie and colleagues [5, 6] showed that the double product reserve (DPR) was a better predictor of mortality than metabolic equivalents, maximal heart rate (HR), systolic blood pressure or HR recovery in 1,655 normal men referred for assessment of exercise capacity. To our knowledge, no previous studies have compared the prognostic value of the DPR against traditional predictors of mortality derived from metabolic gas exchange in a heart failure population. The aim of our study was to evaluate the relative prognostic significance of the DPR in patients with CHF.

\section{METHODS}

The Hull and East Riding Ethics Committee approved the study, and all patients provided informed consent prior to the study. We recruited consecutive patients referred to a com

*Address correspondence to this author at the Carnegie Research Institute, Leeds Metropolitan University, Beckett's Park, Headingley, Leeds, LS6 3QS, UK; Tel: 0113 8123246; E-mail: L.Ingle@ leedsmet.ac.uk munity heart failure clinic with symptoms of breathlessness (NYHA functional class II-III) who were found to have left ventricular systolic dysfunction on investigation. Clinical information obtained included past medical history and drug and smoking history. Clinical examination included assessment of body mass index (BMI), heart rate, rhythm, and blood pressure. Patients were excluded if they were unable to walk without assistance from another person (not including mobility aids), or if they were unable to exercise because of non-cardiac limitations such as osteoarthritis or respiratory disease defined as a predicted $\mathrm{FEV}_{1}<70 \%$.

Heart failure (HF) was defined as the presence of current symptoms of HF, or a history of symptoms controlled by ongoing therapy, due to cardiac dysfunction and in the absence of any more likely cause [7]. Left ventricular function was determined from 2D echocardiography and was carried out by one of three trained operators. Left ventricular function was assessed by estimation on a scale of normal, mild, mild-to-moderate, moderate, moderate-to-severe, and severe impairment and was assessed by a second operator blind to the assessment of the first; where there was disagreement on the severity of left ventricular (LV) dysfunction, the echocardiogram was reviewed jointly with the third operator and a consensus reached. Left ventricular ejection fraction (LVEF) was calculated using the Simpson's formula from measurements of end-diastolic and end-systolic volumes on apical 2D views, following the guidelines of Schiller and colleagues [8] and LVSD was diagnosed if LVEF was $\leq 45 \%$. 
Patients underwent a symptom-limited, treadmill-based maximal CPET using the Bruce protocol modified by the addition of a Stage $0\left(2.74 \mathrm{~km} \cdot \mathrm{h}^{-1}\right.$ and $0 \%$ gradient $)$. Metabolic gas exchange was measured with an Oxycon Delta metabolic cart (VIASYS Healthcare Inc., Philadelphia, PA). Peak oxygen uptake $\left(\mathrm{pVO}_{2}\right)$ was calculated as the average $\mathrm{VO}_{2}$ for the final 30s of exercise. The ventilatory anaerobic threshold (VAT) was calculated by the V-slope method [9]. The $\mathrm{VE} / \mathrm{VCO}_{2}$ ratio was calculated by linear regression analysis using data acquired from the whole test. The peak respiratory exchange ratio (pRER) was calculated as the mean $\mathrm{VCO}_{2} / \mathrm{VO}_{2}$ ratio for the final 30 s of exercise. Blood pressure (BP) was measured using an automated blood pressure monitor (SunTech Tango, USA). Resting BP and heart rate (HR) were measured following 10 min of rest in a supine position. The DPR was calculated as the product of peak systolic blood pressure and peak heart rate subtracted from the product of resting systolic blood pressure and resting heart rate values.

\section{Statistical Analysis}

SPSS (version 14.0) was used to analyse the data. Continuous variables are presented as mean $\pm \mathrm{SD}$, and categori- cal data are presented as percentages. Continuous variables were assessed for normality by the Kolmogorov-Smirnov test. We used Pearson correlation coefficients to calculate association between variables. An independent samples $t$-test was used to calculate differences between alive and deceased patients at 12 months. An arbitrary level of 5\% statistical significance was used throughout (two-tailed).

All survivors were followed for a minimum of 12 months and we therefore give the probability of 12-month survival. All baseline variables (Table 1) were entered as potential univariate predictors of mortality using Cox analysis. Model building was based on backwards elimination $(P$-value for entry was $<0.05 ; P$-value for removal $>0.1)$. A multivariate Cox proportional hazards model using backwards elimination was used to identify independent predictors of all-cause mortality from the remaining univariate predictors. Our outcome measure was all-cause mortality.

\section{RESULTS}

363 patients (mean \pm SD; age $74 \pm 11$ years; $81 \%$ males; LVEF $34 \pm 6 \%$; peak $\mathrm{VO}_{2} 19.0 \pm 5.1 \mathrm{~mL} \cdot \mathrm{kg}^{-1} \cdot \mathrm{min}^{-1} ; \mathrm{VE} / \mathrm{VCO}_{2}$ slope $37 \pm 9$; DPR 10,510 $\pm 6,046 \mathrm{mmHg} \cdot$ beat $^{-1}$ ) were in-

Table 1. Clinical Characteristics of Patients with CHF

\begin{tabular}{|c|c|c|c|}
\hline Variable $($ mean \pm SD $)$ & Alive at 1 Year & Dead at 1 Year & $P$-Value \\
\hline$N$ & 311 & 52 & - \\
\hline Age (years) & $73(11)$ & $74(11)$ & 0.69 \\
\hline BMI $\left(\mathrm{kg} \cdot \mathrm{m}^{-2}\right)$ & $28(5)$ & $26(5)$ & 0.10 \\
\hline $\mathrm{pVO}_{2}\left(\mathrm{~mL} \cdot \mathrm{kg}^{-1} \cdot \mathrm{min}^{-1}\right)$ & $19.6(5.0)$ & $16.1(4.4)$ & $0.0001 *$ \\
\hline $\mathrm{VE} / \mathrm{VCO}_{2}$ slope (full) & $38.0(9.0)$ & $38.8(9.7)$ & 0.86 \\
\hline $\mathrm{AT}\left(\mathrm{mL} \cdot \mathrm{kg}^{-1} \cdot \mathrm{min}^{-1}\right)$ & $18.1(6.8)$ & $11.1(3.5)$ & 0.40 \\
\hline pRER & $1.00(0.11)$ & $1.03(0.12)$ & 0.11 \\
\hline Heart rate reserve & $52(23)$ & $48(31)$ & 0.29 \\
\hline SBP (rest) (mmHg) & $135(25)$ & $130(22)$ & 0.15 \\
\hline SBP (peak) (mmHg) & $163(34)$ & $153(33)$ & $0.049^{*}$ \\
\hline Systolic BP reserve & $28(28)$ & $23(28)$ & 0.27 \\
\hline DBP (rest) (mmHg) & $84(14)$ & $80(21)$ & 0.15 \\
\hline DBP (peak) (mmHg) & $92(22)$ & $85(21)$ & $0.045^{*}$ \\
\hline DPR (mmHg.beat $\left.{ }^{-1}\right)$ & $10,704(5,945)$ & $9,348(6,563)$ & 0.14 \\
\hline
\end{tabular}

BMI: body mass index; LVEF: left ventricular ejection fraction; $\mathrm{pVO}_{2}$ : peak oxygen uptake; ACE-I: ACE-inhibitor; RHR: resting heart rate; PHR: peak heart rate; SBP: systolic blood pressure; DBP: diastolic blood pressure; pRER: peak respiratory exchange ratio; DPR: double pressure reserve; AT: anaerobic threshold; $*$ Significant difference, $P<0.05$ 
DPR (mmHg beat $\left.{ }^{-1}\right)$

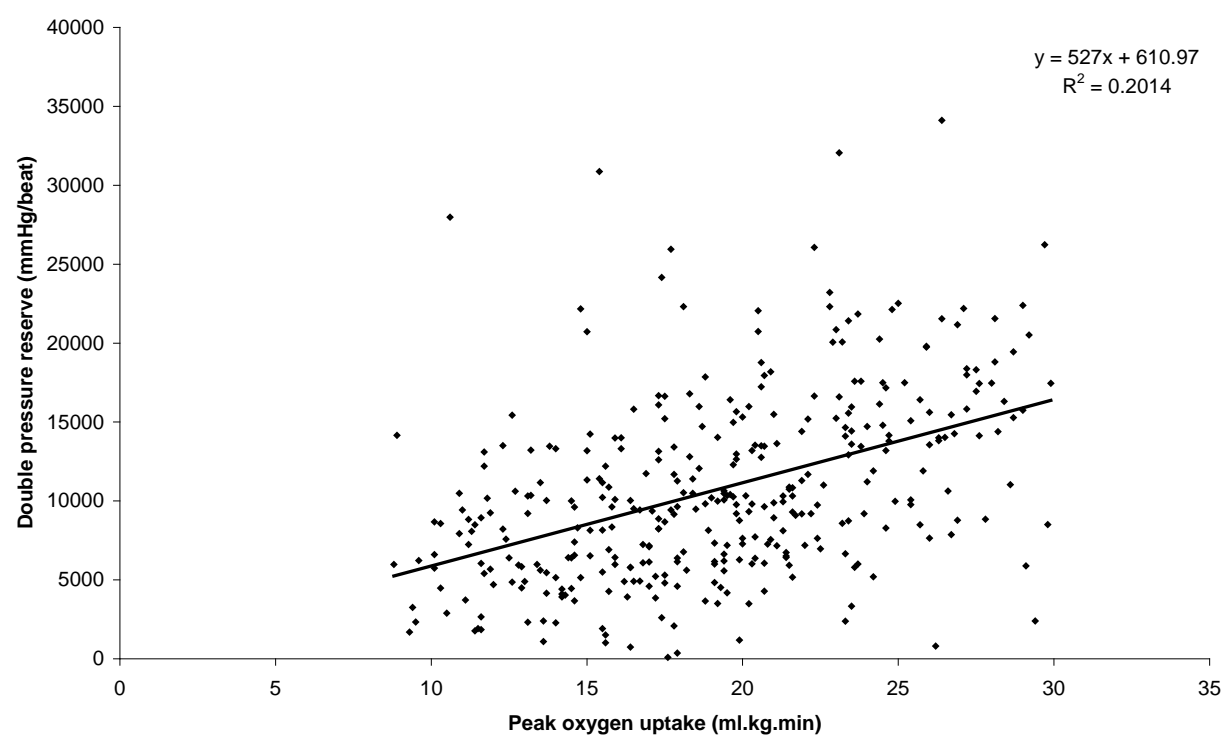

Fig. (1). Relation between peak oxygen uptake and double pressure reserve in CHF.

cluded in the study. Of these, $78 \%$ were prescribed ACEinhibitors, $67 \%$ beta-blockers, and $73 \%$ loop diuretics. Table 1 shows the clinical characteristics of patients included in the study.

Patients who had deceased at 12 months, had lower peak oxygen uptake, exercise duration, peak systolic BP, and peak diastolic BP than alive patients $(P<0.05$; Table 1). There was a weak negative correlation between resting $\mathrm{HR}$ and resting systolic BP $(r=-0.15 ; P=0.004)$, and a weak correlation between heart rate reserve and systolic BP reserve $(r=$ $0.27 ; P=0.0002)$. The DPR was moderately correlated with peak oxygen uptake $(r=0.45 ; P=0.001$; Fig. 1) but showed no association with $\mathrm{VE} / \mathrm{VCO}_{2}$ slope $(r=-0.08 ; P=0.157)$.
During continued follow up, 87 patients died representing a crude death rate of $24 \%$. In surviving patients, the median follow up (inter-quartile range) was 42 (34-50) months. Higher DPR did appear to be related to a better survival, but this effect did not reach statistical significance. By contrast, Kaplan-Meier curves for peak oxygen uptake show a greater separation between quartile ranges $\left(\log\right.$ rank $\chi^{2}=27 ; P=$ 0.001) than for DPR (log rank $\chi^{2}=9 ; P=0.65$ ) (Figs. $2 \&$ 3).

All the variables in Table $\mathbf{1}$ were included as potential candidate univariate predictors of outcome in a Cox model. Six variables met the inclusion criterion $(P<0.1)$; peak $\mathrm{VO}_{2}$, $\mathrm{VE} / \mathrm{VCO}_{2}$ slope, $\mathrm{LVEF}$, age, heart rate (rest), BMI (Table 2)

\section{Survival (\%)}

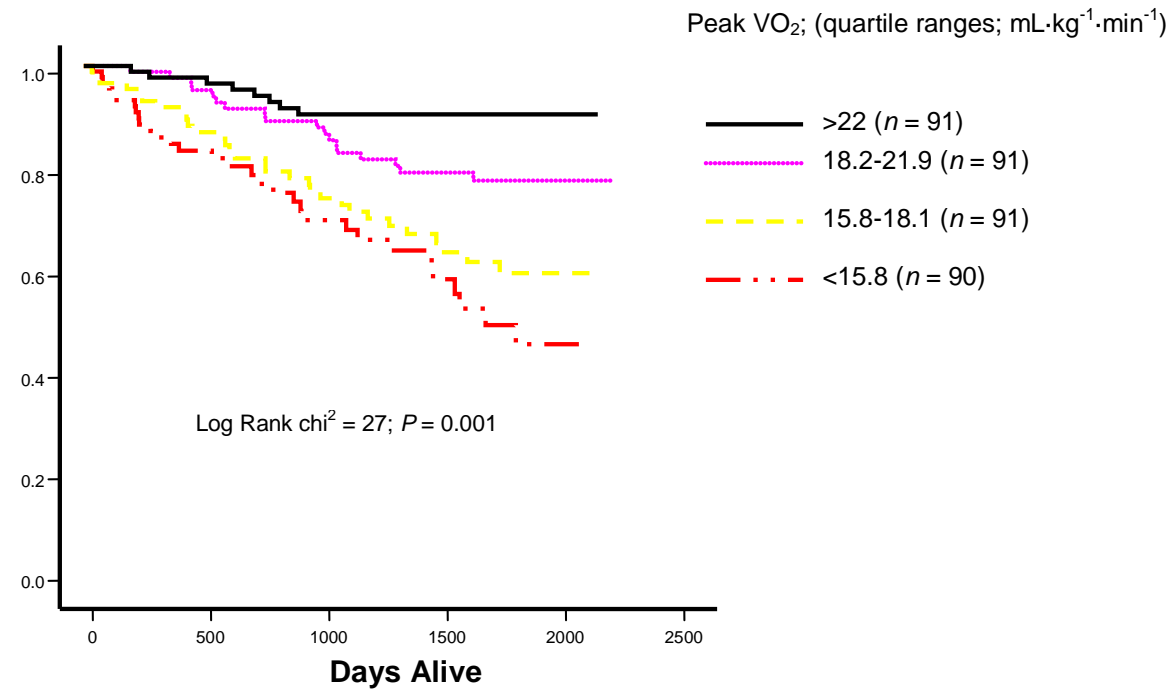

Fig. (2). Kaplan-Meier curve showing prognostic value of peak oxygen uptake in CHF. 


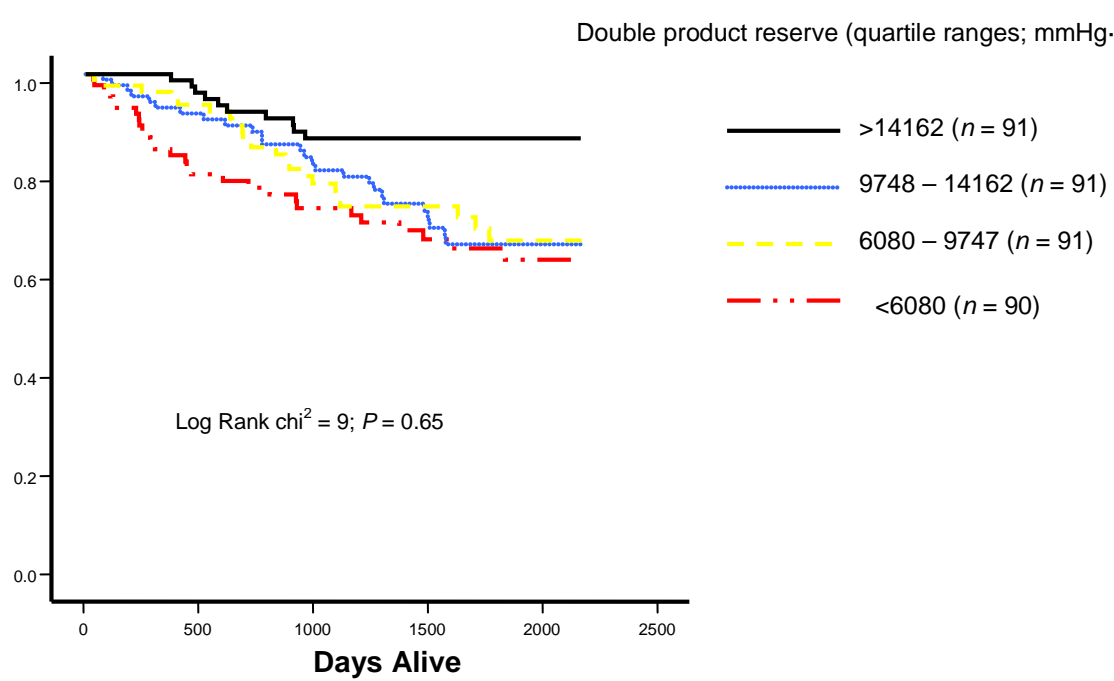

Fig. (3). Kaplan-Meier curve showing prognostic value of the double pressure reserve in CHF.

and were included in the Cox multivariate regression model. DPR was not a univariate predictor of mortality $(P=0.7$; $\mathrm{HR}=1.0 ; 95 \% \mathrm{CI}=0.99-1.0)$. The strongest predictors of allcause mortality was peak oxygen uptake $(\mathrm{HR}=0.87$; $P<0.001 ; 95 \% \mathrm{CI}=0.75-0.99)$ followed by the $\mathrm{VE} / \mathrm{VCO}_{2}$ slope (Table 3).

\section{DISCUSSION}

The DPR is not an independent predictor of mortality during exercise testing with metabolic gas exchange in patients with CHF. Rafie et al. [6] reported that lower DPR was an independent predictor of cardiovascular mortality in men referred for assessment of potential coronary disease, both in those with established cardiovascular disease and those without. The mean DPR value $(10,510 \pm 6,046$
$\mathrm{mmHg} \cdot$ beat $^{-1}$ ) in our patients was similar to that reported by Rafie [6] in patients with CV disease (10,392 \pm 4,846 $\mathrm{mmHg} \cdot$ beat $^{-1}$ ).

The DPR has been shown to be directly related to exercise capacity (METs) and heart rate recovery (HRR), and a DPR of $\leq 10,000 \mathrm{mmHg} \cdot$ beat $^{-1}$ is a strong and independent predictor of CV mortality [6]. A low DPR is a stronger predictor of an increased risk of death than traditional risk factors such as smoking, diabetes, and exercise-related markers such as maximal HR, HRR, or exercise capacity. However, the authors were not able to measure metabolic gas exchange and thus were not able to compare the value of DPP against known predictors of mortality such as peak oxygen uptake and $\mathrm{VE} / \mathrm{VCO}_{2}$ slope $[5,6,10]$. Further, they did not include

Table 2. Univariate Cox Regression Analysis $(P<0.1$ for Inclusion)

\begin{tabular}{|c|c|c|c|c|}
\hline Variables & P-Value & HR & 95\% CI & $\mathbf{X}^{\mathbf{2}}$ \\
\hline \hline Peak $\mathrm{VO}_{2}$ & $<0.001$ & 0.9 & $0.8-0.9$ & 1.90 .8 \\
\hline VE/VCO ${ }_{2}$ slope & 0.04 & 1.03 & $0.95-0.99$ & 1.06 \\
\hline Age & 0.02 & 0.97 & $0.93-1.01$ & 3.3 \\
\hline LVEF & 0.1 & 0.97 & $0.99-1.02$ & 1.1 \\
\hline Heart rate (rest) & 0.1 & 1.01 & $0.94-1.01$ & 2.8 \\
\hline BMI & 0.1 & 0.98 & \\
\hline
\end{tabular}

$\mathrm{HR}=$ hazard ratio $\mathrm{CI}=$ confidence interval; $\mathrm{X}^{2}=\mathrm{Chi}$-square $\mathrm{LVEF}=$ left ventricular ejection fraction; $\mathrm{BMI}=$ body mass index .

Table 3. Multivariate Cox Regression Analysis

\begin{tabular}{|c|c|c|c|c|}
\hline Variable & $\boldsymbol{P}$-Value & HR & 95\% CI & $\mathbf{X}^{\mathbf{2}}$ \\
\hline \hline Age & 0.09 & 1.02 & $0.98-1.05$ & 3.0 \\
\hline Peak $\mathrm{VO}_{2}$ & $<0.001$ & 0.87 & $0.75-0.99$ & 11.8 \\
\hline VE/ $\mathrm{VCO}_{2}$ slope & 0.04 & 1.03 & $1.00-1.07$ & 4.0 \\
\hline
\end{tabular}


patients with $\mathrm{CHF}$. We have shown that prognostic markers derived from metabolic gas exchange are more important than DPR in patients with CHF. The double (rate-pressure) product has been used as an estimate of the maximal performance of the left ventricle during exercise testing [6], and is an indirect marker of myocardial oxygen consumption [11]. However, it does not have any prognostic value in our cohort of CHF patients.

\section{Limitations}

We only evaluated the prognostic impact of DPR against all-cause mortality. We were unable to assess cause-specific mortality in our subset of CHF patients.

\section{CONCLUSION}

In $\mathrm{CHF}$, the DPR is not an independent predictor of mortality. Traditional prognostic markers derived from metabolic gas exchange including peak $\mathrm{VO}_{2}$ and the $\mathrm{VE} / \mathrm{VCO}_{2}$ slope are more important. We recommend that exercise testing with metabolic gas exchange should be incorporated into clinical practice in order to improve prognostic predictive power in patients with $\mathrm{CHF}$.

\section{REFERENCES}

[1] Mancini DM, Eisen H, Kussmaul W, et al. Value of peak exercise oxygen consumption for optimal timing of cardiac transplantation in ambulatory patients with heart failure. Circulation 1991; 83: $778-86$.
[2] Gitt AK, Wasserman K, Kilkowski C, et al. Exercise anaerobic threshold and ventilatory efficiency identify heart failure patients for high risk of early death. Circulation 2002; 106: 3079-84.

[3] Chua TP, Ponikowski P, Harrington D, et al. Clinical correlates and prognostic significance of the ventilatory response to exercise in chronic heart failure. J Am College Cardiol 1997; 29:1585-90.

[4] Clark AL, Volterrani M, Swan JW, et al. The increased ventilatory response to exercise in chronic heart failure: relation to pulmonary pathology. Heart 1997; 77: 138-46.

[5] Sadrzadeh Rafie AH, Sungar GW, Dewey FE, et al. Prognostic value of double product reserve. Eur J Cardiovasc Prev Rehabil 2008; 15: 541-7.

[6] Rafie AH, Dewey FE, Sungar GW, et al. Age and double product (systolic blood pressure $\mathrm{x}$ heart rate) reserve-adjusted modification of the Duke treadmill score nomogram in men. Am J Cardiol 2009; (in press).

[7] Remme WJ, Swedberg K. Comprehensive guidelines for the diagnosis and treatment of chronic heart failure. Task force for the diagnosis and treatment of chronic heart failure of the European Society of Cardiology. Eur J Heart Fail 2002; 4: 11-22.

[8] Schiller NB, Shah PM, Crawford M, et al. Recommendations for quantitation of the left ventricle by two-dimensional echocardiography. American Society of Echocardiography Committee on Standards, Subcommittee on Quantitation of Two-Dimensional Echocardiograms. J Am Soc Echocardiogr 1989; 2: 358-67.

[9] Beaver WL, Wasserman K, Whipp BJ. A new method for detecting anaerobic threshold by gas exchange. J Appl Physiol 1986; 60: 2020-7.

[10] Rafie AH, Dewey FE, Myers J, et al. Age-adjusted modification of the duke treadmill score nomogram. Am Heart J 2008; 155: 1033 8.

[11] Gobel FL, Norstrom LA, Nelson RR, et al. The rate-pressure product as an index of myocardial oxygen consumption during exercise in patients with angina pectoris. Circulation 1978; 57: 549-56.

() Ingle and Clark; Licensee Bentham Open.

This is an open access article licensed under the terms of the Creative Commons Attribution Non-Commercial License (http://creativecommons.org/licenses/by-nc/3.0/) which permits unrestricted, non-commercial use, distribution and reproduction in any medium, provided the work is properly cited. 